\title{
La rete delle organizzazioni fasciste e le iniziative di propaganda culturale in Francia
}

Le réseau des organisations fascistes et les initiatives de propagande culturelle en France

\section{Francesca Cavarocchi}

\section{OpenEdition}

\section{Journals}

\section{Edizione digitale}

URL: http://journals.openedition.org/cei/5419

DOI: 10.4000/cei.5419

ISSN: 2260-779X

\section{Editore}

UGA Éditions/Université Grenoble Alpes

\section{Edizione cartacea}

ISBN: 978-2-37747-076-1

ISSN: 1770-9571

\section{Notizia bibliografica digitale}

Francesca Cavarocchi, «La rete delle organizzazioni fasciste e le iniziative di propaganda culturale in Francia», Cahiers d'études italiennes [Online], 28 | 2019, online dal 15 février 2019, consultato il 26 mars 2021. URL: http://journals.openedition.org/cei/5419; DOI: https://doi.org/10.4000/cei.5419

Questo documento è stato generato automaticamente il 26 mars 2021

(C) ELLUG 


\section{La rete delle organizzazioni fasciste e le iniziative di propaganda culturale in Francia}

Le réseau des organisations fascistes et les initiatives de propagande culturelle en France

Francesca Cavarocchi

\section{Il fascismo e l'organizzazione del consenso fra gli emigrati}

1 Il regime fascista dedicò alla Francia un'attenzione centrale nella sua politica di promozione all'estero; la strategia italiana combinò dagli anni Venti diversi livelli di intervento, il cui tenore e la cui efficacia furono strettamente intrecciati al cangiante andamento delle relazioni diplomatiche bipartite ${ }^{1}$. Si affermò, già a partire dalla metà del decennio, l'esigenza di rispondere all'iniziativa dei fuorusciti, che proprio dall'avamposto parigino avevano iniziato a diffondere analisi e documenti volti a illustrare l'accelerazione autoritaria e i caratteri inediti della dittatura ${ }^{2}$. Con la stabilizzazione del regime, a questa esigenza difensiva si affiancò la formulazione di una più ambiziosa strategia di penetrazione culturale; essa intendeva promuovere un'immagine rassicurante dell'esperimento fascista e delle sue 'realizzazioni', con l'obiettivo di suscitare le simpatie dell'opinione pubblica e di influenzare la politica estera francese in senso filoitaliano.

2 Nello stesso tempo il governo intraprese una precoce iniziativa volta alla fascistizzazione degli emigrati residenti nell'esagono. È difficile fornire una stima precisa delle dimensioni di questa comunità, dati i limiti delle rilevazioni. Secondo $i$ censimenti francesi, la popolazione italiana aumentò da circa 420000 persone nel 1921 (cifra pressoché equivalente a quella del 1911) a 808000 nel 1931; nel 1936, a causa della crisi economica, si registrerà un calo della presenza ufficiale, che passerà a 720000 
unità ${ }^{3}$. Secondo il Commissariato per l'emigrazione italiano, già nel 1924 essa ammontava invece a più di un milione; tale ordine di grandezza sembra più attendibile, date la presenza di una quota di clandestini e le imperfezioni nel sistema di identificazione degli immigrati. Si trattava in ogni caso del gruppo nazionale più forte sul suolo francese, su un totale di circa tre milioni di stranieri, nonché di gran lunga della più ampia collettività di origine italiana nel continente europeo, seguita dalla Svizzera ${ }^{4}$. Sui processi di integrazione nella società francese, nonché sui fenomeni di politicizzazione nei due opposti campi fascista e antifascista, è da tempo disponibile una consistente produzione storiografica, che si è misurata in primo luogo con i contesti regionali in cui più significativa era la presenza di cittadini provenienti dalla penisola ${ }^{5}$.

3 Uno dei primi obiettivi del regime fu il tentativo di assumere il controllo della rete di associazioni già esistenti. Secondo il censimento del Commissariato per l'emigrazione, nel 1923 erano presenti 164 sodalizi: si trattava di società patriottiche, ricreative, di mutuo soccorso, costituite sulla base di solidarietà di mestiere o di appartenenze regionali, per un totale di quasi 20000 iscritti; la Francia, seguita dalla Svizzera, era prevedibilmente il paese europeo in cui questo tessuto era numericamente più consistente e ramificato ${ }^{6}$. La 'conquista' dei circuiti associativi si dipanò dagli anni 1923-1924 fino ai tardi anni Venti con modalità fortemente aggressive e in alcuni casi col ricorso alla violenza; gli emissari del regime arrivarono ad egemonizzare una parte significativa di questi organismi, altri vennero invece conquistati da militanti antifascisti, mentre una minoranza restò autonoma o fu costretta a sciogliersi ${ }^{7}$.

4 Negli stessi anni si assistette anche in Francia alla creazione della rete dei fasci italiani all'estero. Dopo una prima fase di effervescenza, caratterizzata dalla costituzione di sezioni in buona parte spontanea, a partire dal 1928 essi furono posti sotto il controllo della neoistituita Direzione generale degli italiani all'estero (Die) all'interno del ministero degli Esteri. Da diramazione del partito i fasci divennero dunque un'organizzazione posta sotto il controllo statale: tale circostanza significò certo un passaggio dal dinamismo e dal volontarismo delle origini ad una 'normalizzazione', ma pose i circoli sotto la protezione delle rappresentanze diplomatiche e consolari, smussandone in buona parte gli eccessi ed attenuando i motivi di tensione con le autorità locali. La rete francese contava nel 193442 fasci, a cui si aggiungevano 131 sezioni locali; la documentazione disponibile restituisce la creazione di ulteriori sezioni negli anni successivi, mentre a partire dal 1938 le adesioni si ridimensionarono notevolmente, a causa delle crescenti tensioni politico-diplomatiche che condussero settori consistenti delle collettività di emigrati a manifestare un atteggiamento più tiepido nei confronti delle organizzazioni italiane ${ }^{8}$.

5 È difficile fornire una stima precisa degli iscritti dato il carattere disorganico e scarsamente affidabile delle informazioni ufficiali; è tuttavia presumibile che essi si aggirassero nella fase di massima espansione intorno alle 15/20 000 persone ${ }^{9}$. I fasci del resto non furono mai concepiti come strumento di mobilitazione di massa: i meccanismi di reclutamento erano intesi a selezionare una ristretta cerchia di militanti disposti a fare opera di propaganda, a fornire l'ossatura per la diffusione all'estero dei simboli e della ritualità fascista, a sostenere una conflittualità diretta con gli esponenti del fuoruscitismo ${ }^{10}$. Come hanno evidenziato gli studi sui casi locali e regionali, dalla fine degli anni Venti la loro diffusione fu affiancata dalla creazione (o fascistizzazione, come si è visto) di una serie di enti collaterali, che erano invece 
destinati all'inquadramento di massa degli emigrati: i gruppi dopolavoristici e sportivi, le filodrammatiche, le scuole e i doposcuola, le sezioni della Gioventù italiana del littorio all'estero, nonché i circoli locali delle associazioni combattentistiche ${ }^{11}$. A partire dalla fine degli anni Venti si assistette alla costruzione di numerose Case d'Italia, concepite come centri di aggregazione e irradiazione della presenza organizzata del regime. La strategia messa in atto mirava ad utilizzare le leve dell'assistenza e della beneficenza per attrarre le collettività di migranti, con l'obiettivo di sottrarli all'influenza dei gruppi antifascisti nonché di contrastarne i processi di integrazione e l'affiliazione alle organizzazioni politiche e sindacali francesi.

6 I circoli esteri delle società combattentistiche erano presenti soprattutto in Francia: l'Associazione nazionale combattenti (Anc) contava già nel 192764 sezioni, per un totale di 4660 iscritti ${ }^{12}$; nel 1936, secondo i dati ufficiali, delle 26 sezioni estere dell'Associazione nazionale mutilati e invalidi di guerra, 10 erano attive nell'esagono ${ }^{13}$. Tali sodalizi si impegnarono in un'articolata attività, che includeva l'intensificazione delle relazioni con le analoghe organizzazioni francesi, nonché la partecipazione a manifestazioni celebrative del comune sacrificio e della solidarietà interalleata. Il controllo fascista delle associazioni di reduci si rivelò un utile strumento di intervento, data la loro natura di luoghi di sociabilità che si erano guadagnati un crisma di rispettabilità anche per il loro preteso carattere neutrale. Ad esse si aggiungeva l'Unione dei garibaldini delle Argonne, presieduta da Camillo Marabini e sorta in contrapposizione all'antifascista Fédération des Garibaldiens de l'Argonne guidata da Sante Garibaldi; pur contando su un numero ristretto di aderenti, il sodalizio ebbe un ruolo centrale nelle strategie di propaganda, costituendo peraltro un significativo capitolo della contesa politica e simbolica attorno all'eredità del garibaldinismo ${ }^{14}$.

7 Particolare attenzione fu riservata dalla Die all'espansione delle scuole e dei doposcuola. Secondo il censimento del 1927 le scuole elementari, gli asili, i doposcuola e i corsi per adulti erano in Francia 56, per un totale di 4037 allievi; il censimento del 1930 registrava una certa flessione degli iscritti (3 156), anche se i dati inviati a Roma risultavano non di rado incompleti. Nel 1939 le scuole e i corsi erano diminuiti a 48 , sia in seguito ad accorpamenti, sia a causa di una contrazione delle adesioni nella cornice delle crescenti tensioni fra i due paesi; in ogni caso i frequentanti risultavano 7 130, di cui circa 5000 iscritti a scuole e doposcuola e la restante quota ai corsi liberi organizzati in special modo dalla Dante Alighieri. Se tali enti erano sussidiati dalla Die ma in genere gestiti da organismi locali, come la stessa Dante, ifasci o opere ecclesiastiche, risultavano attive a quella data 4 scuole regie, dunque a totale carico dello Stato italiano: un istituto di istruzione media a Parigi, due scuole materne ed elementari e un istituto tecnico-commerciale a Marsiglia $^{15}$. Sebbene manchino statistiche ufficiali per buona parte degli anni Trenta, la documentazione disponibile permette di ipotizzare che la propagazione delle scuole e il numero degli iscritti raggiunsero il punto più alto intorno alla metà del decennio. Nonostante i vertici fascisti avessero riservato un ruolo chiave al settore scolastico, la percentuale degli allievi coinvolti risultò certo una ristretta minoranza se comparata alla popolazione di origine italiana, orientata in massima parte a integrare i figli nel sistema di istruzione francese; maggior successo ebbe invece l'istituzione di doposcuola, di cui gli studi sui casi locali segnalano una significativa crescita nel corso degli anni Trenta ${ }^{16}$.

8 Non è agevole tracciare un bilancio relativo alla forza di questa rete organizzativa e alla sua capacità di mobilitare un reale consenso; l'area di immigrati che essa riuscì ad 
attrarre era una minoranza, né risultò realistica nel lungo periodo la strategia di contrasto ai processi di integrazione e naturalizzazione che caratterizzarono sia pure con difficoltà i diversi contesti di accoglienza. Per quanto instabile e cangiante, il bacino che entrò in contatto in specie con i gruppi assistenziali e ricreativi fu tuttavia significativo; l'interazione con gli organismi fascisti, che presuppose differenti livelli di politicizzazione, fu mossa da bisogni diffusi di sostegno economico e di riconoscimento sociale che coinvolsero in special modo famiglie di recente migrazione. La politica fascista perseguì inoltre con una certa efficacia l'obiettivo di contendere il terreno alle organizzazioni antifasciste e di esercitare verso di esse una costante azione di intimidazione; la normalizzazione delle relazioni con le autorità locali, perseguita tenacemente dalle autorità consolari dopo una prima fase di conflitto, sortì inoltre un risultato significativo, dato che fino alla fine degli anni Trenta essi riuscirono a circoscrivere i motivi di frizione con le prefetture dovuti alle frequenti intemperanze dei responsabili dei fasci.

\section{Le strategie promozionali rivolte all'opinione pubblica francese}

9 L'attività culturale e propagandistica in Francia si organizzò dunque dalla metà degli anni Venti in due settori che rimarranno in buona parte autonomi, ovvero l'inquadramento degli emigrati e le iniziative rivolte all'opinione pubblica francese. Se la «Nuova Italia», il settimanale dei fasci italiani in Francia, fu stampato nei primi anni in edizione bilingue, dal 1926 prevalse nettamente l'uso della lingua italiana; tale scelta obbediva ad una più generale differenziazione nelle proposte editoriali, sia perché si ritenne più efficace concepire pubblicazioni specificamente dedicate ai lettori francesi, sia perché la difesa della lingua d'origine era ritenuta essenziale nel tentativo di preservare l'attaccamento all'identità nazionale. Fra questi due campi si ritrovano tuttavia diversi punti di contatto: si assistette infatti alla progressiva formazione di una rete di funzionari, attivisti e intellettuali impegnati in molteplici ruoli e mobilitati su entrambi i versanti. Si trattava di agenti consolari, commissari dei fasci, insegnanti, giornalisti (principalmente corrispondenti delle testate italiane), esponenti dei circuiti dell'emigrazione intellettuale, nonché notabili delle comunità italiane.

10 In Francia come altrove si procedette, a partire dalla riorganizzazione del ministero degli Esteri attuata nel 1927-1928, all'ampliamento delle rappresentanze consolari, nonché all'immissione in ruolo di nuovo personale selezionato anche secondo criteri di affidabilità politica: le sedi consolari nel 1928 erano aumentate a 20, contro le 10 esistenti nel 1919 (8 consolati e 2 viceconsolati). A una maggiore diffusione corrispose un tendenziale rafforzamento di queste strutture, grazie all'invio da parte del ministero di funzionari preposti a varie mansioni; un ruolo significativo nell'organizzazione della propaganda ebbe ad esempio Amedeo Landini, inviato nel 1928 a Parigi come addetto stampa ${ }^{17}$. Alle autorità consolari fu inoltre affidato un più ampio spettro di compiti rispetto alla fase liberale: esse rivestivano un ruolo complesso, che comprendeva la supervisione sui fasci e sulle organizzazioni di inquadramento degli emigranti, la sorveglianza degli ambienti antifascisti e l'analisi degli orientamenti della stampa periodica locale; erano infine incaricate di avvicinare $i$ circuiti intellettuali stabilendo relazioni informali, nonché promuovendo manifestazioni culturali e celebrative dell'amicizia italo-francese. 
11 Uno degli strumenti della diplomazia culturale italiana fu negli anni Trenta la Società nazionale Dante Alighieri. La Dante, fondata a fine Ottocento con una forte connotazione nazionalista e irredentista, a metà degli anni Venti era diffusa soprattutto in Sud America, nel Mediterraneo, in Svizzera, mentre gli unici comitati francesi erano quelli di Nizza e Grenoble. Negli anni successivi si assistette alla creazione di nuove sezioni: nel 1928 erano attive quelle di Avignone, Tolosa, Mentone, Mulhouse e Nancy, mentre nel 1936 i circoli erano diventati 15, affidati alla presidenza dei consoli o di notabili delle colonie italiane ${ }^{18}$.

12 Negli anni Venti le attività della Dante erano principalmente finalizzate a rafforzare il senso di appartenenza all'interno delle comunità di emigrati e si rivolgevano in particolare al ceto intermedio delle colonie. Tale vocazione fu ridefinita intorno al 1930, in corrispondenza con una più diretta fascistizzazione del sodalizio; da questa fase si assistette infatti al tentativo di attrarre il pubblico francese attraverso nuove linee di intervento, ispirate e controllate dalla centrale romana.

13 Il profilo della Dante restò principalmente legato alla propaganda linguistica e culturale. La direzione del circolo di Parigi, fondato nel 1931, fu affidata allo scrittore Lionello Fiumi; nel 1934 Fiumi venne escluso dal consiglio direttivo e sostituito dal garibaldino Marabini, interprete di un orientamento volto a ingaggiare la Dante in una più scoperta attività di propaganda. Tale avvicendamento non incontrò l'approvazione del sottosegretariato alla Propaganda della presidenza del consiglio, che affermò che la società dovesse tenersi lontano dai temi più direttamente politici; le iniziative volte alla propagazione ideologica e alla tessitura di contatti con personalità politiche dovevano essere invece appannaggio di altri enti o di emissari votati ad un lavoro informale ${ }^{19}$.

14 Negli anni Trenta la Dante propose dunque calendari di conferenze, conversazioni letterarie, concerti e intrattenimenti mondani; dal 1934 i programmi delle sezioni furono coordinati grazie alla collaborazione fra la direzione della Società ed il sottosegretariato (poi ministero) della Propaganda, che predisponevano giri di conferenze di intellettuali e accademici italiani nelle diverse sezioni all'estero.

15 Tra i temi proposti in Francia, grande attenzione era offerta alle radici ed ai luoghi simbolici della comune cultura 'latina', con riguardo alla storia della lingua, alla letteratura, nonché alla celebrazione di personalità paradigmatiche dei legami secolari fra i due popoli, quali Petrarca, Mistral, Alfieri e Stendhal. L'illustrazione dei caratteri della «nuova Italia» insisteva soprattutto sulla modernizzazione nei campi dell'organizzazione culturale e artistica e dell'assistenza sociale, sui progressi della ricerca applicata e delle scienze agronomiche; si tentava inoltre di conferire una dignità accademica alla presentazione dei caratteri dell'esperienza corporativa grazie alla partecipazione di teorici come Gino Arias e Robert Michels. Dall'Italia furono inviati letterati e giuristi organici al regime, da Paolo Arcari a Guido Bortolotto, noti specialisti come il soprintendente Achille Bertini Calosso ed il musicista Cesare Valabrega, nonché concertisti già affermati come Ornella Puliti Santoliquido. Sul versante francese, la Dante riuscì a coinvolgere diversi intellettuali italianisants, esponenti di una rete piuttosto ampia che ritroviamo coinvolti in numerose iniziative di amicizia italo-francese: fra gli altri Henri Massis, gli scrittori Gabriel Faure e René Benjamin, l'ex ministro dell'Educazione nazionale Anatole de Monzie, gli accademici di Francia Louis Gillet e Claude Farrère ${ }^{20}$. Si trattava di figure dai differenti percorsi, alcune organiche o vicine all'Action française, altre appartenenti al più ampio campo conservatore, accomunate dall'attrazione verso l'esperimento fascista e dalla 
propensione a legittimare l'Italia come un interlocutore privilegiato della politica estera francese.

Fra le principali linee di intervento della Dante vi fu l'organizzazione di corsi di lingua e cultura italiana destinati al pubblico locale. Un'iniziativa del console di Parigi Camerani condusse nel 1930 alla creazione di una Scuola di cultura, patrocinata dall'Union intellectuelle franco-italienne col concorso della Federazione italiana professionisti ed artisti. La struttura era in realtà finanziata dal ministero delle Corporazioni, che intendeva farne una centrale della propaganda corporativa tra studenti, intellettuali e professionisti parigini ${ }^{21}$. L'Union era un sodalizio informale volto alla propagazione dell'insegnamento della lingua italiana, a cui avevano aderito specialisti francesi e docenti provenienti dalla penisola. Grazie alla collaborazione di Henri Hauvette, promotore dell'associazione e titolare della cattedra di italianistica alla Sorbona, la scuola trovò ospitalità presso l'Istituto di lingue moderne dell'ateneo; al di là delle cifre ufficiali, che attestavano circa 450 alunni nel 1934-1935, essa esemplificava un'offerta culturale in linea con l'iniziativa della Dante, che combinava corsi di lingua, illustrazione del panorama artistico e letterario e storia del giovane Stato unitario. Questa formula aveva l'obiettivo di presentare il regime come un esito salvifico rispetto all'instabilità attraversata dal paese nella congiuntura postbellica e come un sistema originale e dinamico che costituiva la risposta italiana alla crisi politica europea; nelle strategie discorsive esplicite si puntava dunque non tanto a sottolineare l'esportabilità del modello fascista, quanto a legittimarne la funzione storica a partire dalle specifiche condizioni della penisola.

17 Grande attenzione fu riservata dalle rappresentanze diplomatiche in Francia alla costituzione e allo sviluppo dei Comités France-Italie $e^{22}$. Un Comitato nazionale fu ricostituito nel 1929 da un primo nucleo fondato nel 1923; sorto con l'obiettivo di «rafforzare i legami di amicizia» tra i due paesi a prescindere da «questioni politiche o religiose ${ }^{23}$, esso fu concepito come un sodalizio bilaterale. Primo presidente fu Albert Besnard, a cui successe Pierre de Nolhac, mentre vennero nominati vice-presidenti Jean Rivain, Paul Hazard e due esponenti dell'élite italiana residente a Parigi, Ottavio Lanza Branciforte duca di Camastra e l'industriale Carraro. Furono inoltre costituite sette commissioni tematiche: una commissione per le lettere (a cui parteciparono Henry Bordeaux, Gabriel Faure, Tommaso Marinetti e Ugo Ojetti), una «commissione di studiosi» (che contava fra gli altri l'antichista Jérôme Carcopino), una commissione per la storia, presieduta da Louis Madelin, due commissioni dedicate alle belle arti (composte da artisti e studiosi quali Émile Mâle, direttore dell'École française di Roma, e Romano Romanelli, scultore e accademico d'Italia), e infine una commissione per la stampa e una per le scienze pure, con una sezione economica e una medica ${ }^{24}$. Di quest'ultima facevano parte diverse personalità, fra cui il matematico e accademico delle Scienze Émile Picard, Louis Lumière, Maurice de Broglie, Guglielmo Marconi e il chimico Nicola Parravano.

Su iniziativa di diverse personalità (fra le quali il segretario del comitato parigino Philippe de Zara, acceso simpatizzante del fascismo) si osserva fino al 1935-36 la fondazione di 24 comitati locali, come quello di Marsiglia, affidato a Maurice Mignon e allo specialista di letteratura provenzale Émile Ripert, quello di Tolosa, presieduto dal chimico e premio Nobel Paul Sabatier, e la sezione di Bordeaux, costituita per iniziativa del console Zanotti Bianco; nel 1934 fu fondata una sezione a Lione, la cui presidenza onoraria venne conferita a Édouard Herriot ${ }^{25}$. 
19 I Comitati si impegnarono in un'attività che prevedeva diversi livelli di intervento: il sostegno alle iniziative di riavvicinamento diplomatico, l'organizzazione di eventi culturali e celebrativi, la programmazione di viaggi di studio e di amicizia, la promozione di scambi commerciali ${ }^{26}$. Diversi gli incontri dedicati all'illustrazione dei principi del regime, fra i quali nel 1935 una lezione di Giuseppe Bottai sul rapporto tra rivoluzione francese e rivoluzione fascista ${ }^{27}$; si tennero inoltre numerose conferenze bilaterali sul corporativismo e sulle politiche di assistenza e previdenza sociale, nonché sulla 'modernità' raggiunta dalla penisola in vari campi della vita pubblica: anche in questo caso grande attenzione era rivolta alla parola d'ordine della 'latinità', che previde approfondimenti sul contributo dei due paesi al progresso delle scienze teoriche e applicate in collaborazione con varie personalità d'oltralpe, fra le quali il chirurgo Louis Dartigues, presidente dell'Union médicale latine $e^{28}$.

Il Comitato e le sue diramazioni rimasero un organismo bilaterale, fondato principalmente per facilitare il rapprochement franco-italiano, la cui iniziativa si indebolì dopo il 1936; è in ogni caso da sottolineare che le rappresentanze italiane svolsero un ruolo discreto ma efficace di indirizzo, filtrando ad esempio l'invio dall'Italia di specialisti e personalità pubbliche. Il sodalizio vide la partecipazione non solo di figure interessate al riavvicinamento con l'Italia in funzione antitedesca, ma anche di numerosi italianisants affascinati dal modello fascista.

21 La Dante e i Comités furono due fra i principali vettori di una composita strategia di penetrazione che fu elaborata in modo progressivo a partire dalla seconda metà degli anni Venti e che vide l'interazione fra diverse centrali: il ministero degli Esteri, prima attraverso l'ufficio stampa e poi con la creazione della Die, che ebbe giurisdizione soprattutto sull'organizzazione del consenso fra gli emigrati; dal 1934 il sottosegretariato alla Propaganda della presidenza del consiglio, primo nucleo del ministero della Cultura popolare. Si trattò di una strategia non lineare, che implicò conflitti e sovrapposizioni fra i diversi uffici, ma che mobilitò notevoli energie e si concretizzò nella formazione di un network informale ma ampio di collaboratori. Anche dopo la creazione del Minculpop furono in ogni caso gli avamposti diplomatici e consolari a mantenere un ruolo chiave, dato che solo essi disponevano dei necessari strumenti di mediazione e interlocuzione coi circuiti francesi.

Uno dei compiti dei consolati fu quello di coltivare relazioni con i lettori e i professori di lingua e letteratura italiana nelle università francesi. Dal 1930 il ministero degli Esteri italiano sovvenzionò la costituzione di nuovi lettorati, che si affiancarono a quelli finanziati dal ministero della Pubblica istruzione francese; nel 1939 ne risultavano in funzione 13, ad esempio a Digione (dal 1931-1932), Nancy (dal 1932-1933), Bordeaux (dal 1934-1935 ${ }^{29}$. Come ha dimostrato Jérémie Dubois, solo una parte dei lettori risultò effettivamente disponibile a mobilitarsi assumendo funzioni di propaganda; furono in special modo alcune personalità, come Giovan Battista Agnoletti o Giuseppe Gerace, i cui incarichi erano inscritti nel bilancio del ministero italiano, ad impegnarsi in un'attività di promozione della «nuova Italia» negli ambienti accademici e intellettuali locali.

Intorno al 1935, nel clima di avvicinamento sancito dagli accordi fra Mussolini e Laval, il ministero degli Esteri considerò la possibilità di concludere un accordo culturale con la Francia e di impiantare anche a Parigi un istituto di cultura italiana, secondo un modello già seguito in altri paesi europe ${ }^{30}$; il progetto fu successivamente abbandonato sia in considerazione della crisi nei rapporti con la Repubblica dopo la vittoria del 
Fronte popolare, sia perché la creazione di un ente ufficiale nella capitale francese avrebbe comportato un impegno finanziario e organizzativo difficilmente sostenibile; questo passaggio confermò che la costruzione di una rete policentrica si era rivelata la scelta più appropriata nel contesto delle cangianti e delicate relazioni diplomatiche fra i due paesi.

\section{La mobilitazione dei networks intellettuali e artistici italo-francesi}

Tale strategia, ispirata dallo stesso Mussolini, aveva puntato fin dagli anni Venti ad attivare i tradizionali réseaux intellettuali e artistici franco-italiani, caratterizzati da intense e solide collaborazioni. Rilevante fu l'iniziativa nel campo delle arti figurative, grazie anche alla mobilitazione di artisti italiani residenti a Parigi o fortemente legati ai circuiti francesi. Enrico Prampolini, Carlo Carrà, Mario Tozzi, collaboratori della «Nuova Italia» e del fascio parigino, furono fra i principali mediatori all'interno di queste reti; essi si impegnarono in una serie di iniziative promozionali nonché nella realizzazione di vari eventi espositivi, come l'importante mostra d'arte italiana organizzata nel 1933 alla Galerie Charpentier col patrocinio dell'ambasciatore italiano e del ministro de Monzie ${ }^{31}$. Nonostante le fortune del futurismo in Francia risultassero da tempo in fase calante, Marinetti rimase l'esponente più visibile dell'arte italiana a Parigi fino alla prima metà degli anni Trenta, il personaggio a cui il regime affidò un più diretto ruolo di rappresentanza, non privo di un crisma di ufficialità ${ }^{32}$. Egli intervenne su invito dei Comités France-Italie o come delegato del governo italiano a diversi eventi pubblici, come le celebrazioni virgiliane e mistraliane del 1930; partecipò infine alla cerimonia inaugurale della Mostra d'arte italiana tenutasi il 16 maggio 1935 alla presenza di Lebrun. L'esposizione parigina segnò il culmine dell'iniziativa italiana nel settore delle arti figurative, collocandosi nella fase di più intenso riavvicinamento diplomatico fra i due paesi; patrocinata dai comitati France-Italie e Italia-Francia, la sua organizzazione vide il coinvolgimento del segretario della Biennale di Venezia Antonio Maraini e dello stesso sottosegretariato alla Propaganda diretto da Ciano ${ }^{33}$. Negli anni precedenti numerosi erano stati gli eventi posti sotto il patrocinio delle autorità italiane, dalla partecipazione al Salon international du livre d'art ospitato nel 1931 al Petit Palais fino agli imponenti padiglioni allestiti nello stesso anno all'Exposition coloniale internationale di Parigi ${ }^{34}$.

Fin dagli anni Venti avevano assunto un ruolo significativo le iniziative in campo editoriale, con l'obiettivo di diffondere un articolato spettro di pubblicazioni rappresentative della «nuova Italia». La promozione di traduzioni, attraverso collaborazioni e accordi con case editrici francesi, fu perseguita grazie all'attivazione di una rete informale di mediatori culturali, in grado di interloquire con i circuiti letterari e critici di italianisants. L'iniziativa del già citato Lionello Fiumi è esemplificativa di questa traiettoria: Fiumi, fra i principali animatori della sezione parigina della «Dante Alighieri», diresse la rivista «Dante», pubblicata dal 1932 al 1940 (e dal'34 resasi indipendente dall'associazione). Il periodico si distinse per una notevole attività traduttiva, volta a presentare autori fino ad allora inediti, e per un impianto ideologico attento a rivendicare il primato della latinità ed i nessi profondi fra le tradizioni culturali romanze; un orientamento che sottendeva tuttavia una non esibita ma sostanziale inclinazione nazionalista ed una convergenza con la politica culturale del 
regime $^{35}$. In questa cornice ebbero un ruolo rilevante italianisti e traduttori come Maurice Mignon, Eugène Marsan, Eugène Bestaux, Benjamin Crémieux, Alfred Mortier; la loro collaborazione facilitò la circolazione di testi rappresentativi della nuova temperie culturale postbellica, l'accesso a case editrici di rilievo nazionale, la pubblicazione di contributi e recensioni su riviste culturali e testate quotidiane. Si dovettero a questa rete la promozione in lingua francese dell'opera di Pirandello e della più recente letteratura italiana, ma anche la mediazione di testi di attualità politica, dal diario di guerra di Mussolini alla biografia del maresciallo Italo Balbo a cura di Antonio Aniante $^{36}$.

Per quanto riguarda l'editoria di settore, è da segnalare il ruolo dell'Ente nazionale per le industrie turistiche, che inaugurò nel 1926 una nuova sede a Parigi e che strinse accordi con agenzie e librerie locali; l'ente distribuì una mole rilevante di pubblicazioni, guide gratuite e materiale promozionale in lingua francese, dimostrando un significativo dinamismo nel settore della propaganda turistica ${ }^{37}$.

I consoli svilupparono un'attività di collegamento tra Roma e le reti attive in Francia, nonché di controllo e mediazione fra i diversi attori sul campo; promossero inoltre la costituzione di biblioteche di cultura italiana, l'invio di pubblicazioni, la formazione di un archivio di personalità interessate a ricevere documentazione sull'Italia fascista. Un particolare impegno fu riservato alla diffusione di articoli, informazioni e materiali fotografici ad agenzie di stampa e periodici politici, culturali e di settore; tale iniziativa, coordinata dalla metà degli anni Trenta dal ministero della Propaganda, fu dispiegata grazie alla tessitura di contatti con editori e giornalisti francesi ${ }^{38}$.

\section{La strategia fascista tra soft power e propaganda politica}

Sono stati in parte ricostruiti i contatti fra il regime ed esponenti delle formazioni di estrema destra come i Francisti di Bucard, le Jeunesses patriotes e successivamente il Partito popolare francese di Doriot. Ad avvicinare i responsabili dei gruppi francesi furono in primo luogo i Comitati d'azione per l'universalità di Roma, organismo sorto nei primi anni Trenta con l'obiettivo di sviluppare più intense forme di cooperazione con i movimenti europei; svolsero un ruolo in questo campo anche dirigenti della rete dei fasci e altri emissari ${ }^{39}$. Sarebbe tuttavia utile indagare con uno sguardo più ampio questa trama di relazioni: una lettura attenta alle connessioni transnazionali fra movimenti e partiti di destra e filofascisti ha infatti da tempo evidenziato la circolarità di modelli, pratiche, parole chiave, a prescindere dalle molteplici traiettorie nazionali che ne segnarono l'irriducibile pluralità. Si tratta di una prospettiva che ha contribuito ad arricchire e muovere i quadri di interpretazione, introducendo elementi nuovi rispetto alla lunga controversia sul livello di «impregnazione» fascista che caratterizzò le diverse formazioni francesi e sul reale peso specifico da esse assunto negli anni Trenta $^{40}$.

È utile in questa sede sottolineare che Roma non rinunciò certo a perseguire anche questo canale di penetrazione, ma prevalse un orientamento inteso a operare in forma discreta, sia per evitare irrigidimenti e accuse di sconfinamento, sia perché i vertici fascisti manifestarono riserve sulla tenuta e l'affidabilità politica delle formazioni fascistizzanti. Tali indicazioni, di cui si fecero interpreti le rappresentanze diplomatiche, furono recepite non senza incidenti e contraddizioni fra le varie agenzie 
impegnate sul campo: era infatti diffusa fra i dirigenti dei fasci francesi la propensione a intraprendere forme più scoperte e agitatorie di propaganda. Un investimento prioritario fu invece dedicato a una strategia di penetrazione indiretta e rivolta ad un pubblico largo, che puntava alla promozione dell'immagine del regime nei circuiti intellettuali e nei settori d'opinione più sensibili al tema del «ritorno all'ordine». Fra le personalità coinvolte nella rete di collaborazioni si ritrovano sia diversi intellettuali provenienti dall'esperienza dell'Action française, sia esponenti di un più ampio spettro di posizioni conservatrici e fautori di un modello 'forte' rispetto al parlamentarismo della III Repubblica. L'iniziativa fascista fu da questo punto di vista non priva di risultati, contribuendo prima alla legittimazione e poi alla diffusione di un'immagine 'rispettabile' del regime, con l'obiettivo di influenzare in senso filo-italiano la politica estera francese, nonché di svolgere sottotraccia una costante azione di disturbo nel dibattito pubblico interno. Le reti di amicizia franco-italiane furono messe alla prova durante la campagna antisanzionista nel 1935-1936, che segnò una forte mobilitazione delle centrali attive in Francia con l'obiettivo di allentare il fronte filosocietario e di suscitare reazioni pubbliche di sostegno al regime: il noto manifesto «des intellectuels français pour la défense de l'Occident», promosso da Massis e pubblicato nell'ottobre 1935, fu dunque un'iniziativa autonoma, ma preparata da una fitta trama di relazioni rafforzata negli anni precedenti ${ }^{41}$.

\section{Conclusioni}

Le iniziative di propaganda culturale affidarono un ruolo centrale all'illustrazione dei caratteri della modernizzazione 'dall'alto' attuata dal regime, con particolare riguardo all'organizzazione corporativa, all'assistenza sociale, ai grandi progetti urbanistici, alla vivacità della produzione artistica. Esse intendevano veicolare lo scenario di un paese in trasformazione, promuovendo un'immagine di dinamismo ed esuberanza, ma anche di stabilità e forte coesione interna; di questo equilibrio si faceva garante la dittatura, che aveva rappresentato un argine rispetto alla crisi postbellica e che si avviava a superare i mali antichi della penisola. Prevalse dunque una rappresentazione conciliante dell'esperienza fascista, che puntava a presentare il governo italiano come un interlocutore affidabile, depotenziandone agli occhi del pubblico francese i tratti revisionisti e aggressivi. L'evocazione della «solidarietà latina» e di tratti identitari comuni in contrapposizione all'Europa germanica e «nordica» fu un elemento costante di questa strategia discorsiva: essa ricorse ad un campo semantico che aveva trovato proprio in Francia una sua articolata elaborazione e che aveva costituito uno dei principali vettori degli scambi letterari e artistici franco-italiani fin dal primo Novecento ${ }^{42}$. E' utile sottolineare la discrasia fra l'uso che della categoria si faceva nella propaganda interna, in cui l'idea del campo latino era strettamente intrecciata alla rivendicazione del primato italiano ed era connotata da una più o meno esplicita valenza antifrancese, rispetto all'accezione che i mediatori culturali ne promossero in Francia, attenta evidentemente a rimarcare i tratti comuni nella tradizione civile e 'spirituale' dei due paesi ${ }^{43}$.

Le attività di propaganda e le agenzie culturali italiane in Francia mantennero nel corso del ventennio una dimensione plurale. Questa molteplicità di attori rispondeva a diverse esigenze, dalla costruzione del consenso fra gli emigrati alla promozione dell'immagine del regime presso settori differenziati dell'opinione pubblica; la trama 
organizzativa faticosamente costruita si dimostrò piuttosto efficace, dato che riuscì almeno in parte a mobilitare gli ampi circuiti intellettuali italo-francesi e a garantire al regime uno spazio di iniziativa politico-diplomatica in aperta competizione con il fronte antifascista. Questa strategia raggiunse i risultati più visibili nella prima metà degli anni Trenta, quando più intensi furono i tentativi di approdare a una conciliazione fra gli interessi dei due paesi, arretrò con la vittoria del Fronte Popolare, ma fu definitivamente superata nel 1938-1939; l'Italia aveva ormai chiuso ogni residuo canale di interlocuzione con la Repubblica, mentre le restrizioni varate dal governo francese condussero allo scioglimento ufficiale delle organizzazioni fasciste.

\section{NOTE}

1. Sulle relazioni italo-francesi fra le due guerre si vedano almeno J.-B. Duroselle e E. Serra (a cura di), Italia e Francia dal 1919 al 1939, Milano, Ispi, 1981; W. Shorrock, From Ally to Enemy. The Enigma of Fascist Italy in French Diplomacy. 1920-1940, Kent (Ohio), Kent State University Press, 1988; P. Guillen, Franco-Italian Relations in Flux, 1918-1940, in R. Boyce (a cura di), French Foreign and Defence Policy, 1918-1940. The Decline and Fall of a Great Power, London-New York, Routledge, 1998, pp. 149-163; J.-M. Palayret, L'alliance impossible. Diplomatie et outil militaire dans les relations francoitaliennes, Vincennes, Service historique de la marine, 2004.

2. Fra le principali pubblicazioni, che si affiancavano a un'articolata presenza sulla stampa periodica, L. Sturzo, L'Italie et le fascisme, Parigi, Alcan, 1927; S. Trentin, L'aventure italienne. Légendes et réalités, Parigi, PUF, 1928; G. Salvemini, La terreur fasciste, 1922-1926, Parigi, Gallimard, 1930; per un inquadramento recente e una bibliografia sul tema, si rimanda a A. Bechelloni, Esilio e antifascismo, «Parolechiave», $\mathrm{n}^{\circ}$ 1, 2009, pp. 141-157; ancora utile S. Tombaccini, Storia dei fuorusciti italiani in Francia, Milano, Mursia, 1988.

3. M. Amar e P. Milza, L'immigration en France au Xx siècle, Parigi, Colin, 1990, p. 186; cfr. anche P. George, L'immigration italienne en France de 1920 à 1939: aspects démographiques et sociaux, in P. Milza (a cura di), Les Italiens en France de 1914 à 1940, Roma, École française de Rome, 1986, pp. 45-67.

4. Annuario statistico dell'emigrazione italiana. Dal 1875 al 1925, Roma, Commissariato generale dell'emigrazione, 1926, p. 1534.

5. Per un inquadramento, cfr. almeno P. Milza (a cura di), Les Italiens en France, cit.; A. Bechelloni, M. Dreyfus e P. Milza (a cura di), L'intégration italienne en France, Bruxelles, Complexe, 1995. Sull'organizzazione fascista in Francia, C. Wiegandt-Sakoun, Le fascisme italien en France, in P. Milza (a cura di), Les Italiens en France, cit., pp. 421-469; P. Milza, Le fascisme italien en France (1938-1943), in G. Perona (a cura di), Gli italiani in Francia 1938-1946, «Mezzosecolo», vol. 9, 1994, pp. 91-104; E. Vial, I Fasci in Francia, in E. Franzina e M. Sanfilippo (a cura di), Il fascismo e gli emigranti, Roma-Bari, Laterza, 2003, pp.27-42; R. Schor, Les immigrés italiens en France et l'engagement fasciste, 1922-1939, «Parlement[s]. Revue d'histoire politique», $\mathrm{n}^{\circ}$ 3, 2011, pp. 130-140, disponibile online all'indirizzo <www.cairn.info/revue-parlements1-2011-3-page-130.htm>. Fra i principali lavori su singole regioni, P. Milza, Le fascisme italien à Paris, «Revue d'histoire moderne et contemporaine», vol. 30, $\mathrm{n}^{\circ} 3,1983$, pp. 420-452; F. Cavarocchi, Propaganda e associazionismo fascista nelle comunità di emigrazione: il caso di Parigi (1922-1939), «Società e storia», $\mathrm{n}^{\circ} 2,2008$, pp. 279-307; R. Damiani, Les Italiens dans le Nord et le Pas-De-Calais entre 1919 et 1939, in P. Milza (a 
cura di), Les Italiens en France, cit., pp. 633-659; P. Videlier, Les Italiens de la région lyonnaise, in P. Milza (a cura di), Les italiens en France, cit., pp. 661-691; R. Schor, Il fascismo italiano nelle AlpesMaritimes, 1922-1939, «Notiziario dell'Istituto della Resistenza in Cuneo e provincia», $\mathrm{n}^{\circ} 26,1984$, pp. 21-57; É. Temime, Les Italiens dans la région marseillaise pendant l'entre-deux-guerres, in P. Milza (a cura di), Les Italiens en France, cit., pp. 547-575; sul Sud-Ovest, C. Maltone, Les Associations fascistes italiennes dans le Sud-Ouest de la France, in C. Maltone e M. Rouch (a cura di), Sur le pas des Italiens en Aquitaine au XX siècle, Talence, MSHA, 1998, pp. 115-150; L. Teulières, Immigrés d'Italie et paysans de France, 1920-1944, Toulouse, Presses universitaires du Mirail, 2002; sul Sud-Ovest e la Lorena, P. Pinna, Migranti italiani tra fascismo e antifascismo. La scoperta della politica in due regioni francesi, Bologna, CLUEB, 2012; sulla Lorena, D. Francfort, Être mussoliniens en Lorraine : les fascistes italiens face aux associations 1921-1939, «Revue d'histoire moderne et contemporaine», vol. 38, $\mathrm{n}^{\circ} 2$, 1991, pp. 313-336.

6. Commissariato generale dell'emigrazione, Censimento della popolazione italiana all'estero nel 1924, vol. 2, f. 1, Roma, Tipografia delle cartiere centrali (poi Sandron), 1924-1925, p. 133.

7. Cfr. ad es. F. Cavarocchi, Propaganda e associazionismo fascista, cit., pp. 291-295; P. Pinna, Migranti italiani tra fascismo e antifascismo, cit., pp. 280-314 ; L. Teulières, Immigrés d'Italie, cit., p. 167.

8. Per i dati del 1934, P. Parini, Gagliardetti italiani nel mondo, Novara, De Agostini, 1934, cit. in C. Wiegandt-Sakoun, Le fascisme italien en France, cit., p. 441. Sulla fase 1938-1939, P. Milza, Le fascisme italien en France (1938-1943), cit.; L. Rapone, Les Italiens en France, un problème pour la politique étrangère italienne, entre guerre fasciste et retour à la démocratie, in P. Milza e D. Peschanski (a cura di), Exils et migrations. Italiens et espagnols en France 1938-1946, Parigi, L'Harmattan, 1994, pp. 175-197.

9. Pierre Milza ha avanzato una stima di 12000 iscritti per gli anni 1937-1938, in cui tuttavia le adesioni risultavano in fase calante (P. Milza, Voyage en Ritalie, Parigi, Plon, 1993, p. 249).

10. Per un'analisi dei meccanismi di reclutamento dei fasci mi permetto di rimandare a F. Cavarocchi, La sorella latina. La diplomazia culturale fascista in Francia e in Germania, di prossima pubblicazione.

11. Cfr. n. 5 .

12. C. Maltone, Les Associations fascistes italiennes, cit., p. 121. Sulle associazioni combattentistiche nel Sud-Ovest e in Lorena, P. Pinna, Migranti italiani tra fascismo e antifascismo, pp. 250-280.

13. Almanacco degli italiani all'estero. 1936-XIV, Roma, Edizioni Roma, p. 121.

14. Sul tema si rimanda a N. Violle, Garibaldiens de l'Argonne, soldats du souvenir et messagers de l'Italie Nouvelle, «Italies», $\mathrm{n}^{\circ} 19, \quad 2015$, consultabile online all'indirizzo <http:// journals.openedition.org/italies/5217>.

15. Cfr. Ministero degli Affari esteri, Direzione generale delle scuole italiane all'estero, Annuario delle scuole italiane all'estero. 1927, Roma, Libreria del Provveditorato generale dello Stato, 1928; Ministero degli Affari esteri, Direzione generale degli italiani all'estero e scuole, Annuario delle scuole italiane all'estero. 1930, Roma, Tip. del Ministero degli affari esteri, 1930; Ministero degli affari esteri, Direzione generale degli italiani all'estero, Annuario delle scuole e delle istituzioni culturali italiane all'estero. 1939-1940, Roma, Ministero degli affari esteri, 1942.

16. Cfr. ad es. F. Cavarocchi, Propaganda e associazionismo fascista, cit., pp. 301-306; C. Maltone, Les Associations fascistes italiennes, cit., pp. 134-135.

17. «Elenchi del personale», Roma, Tip. del Ministero degli Esteri, 1922-1939.

18. Almanacco degli italiani all'estero, cit., p. 110. Sulla storia della Dante nel periodo fascista, cfr. P. Salvetti, Immagine nazionale ed emigrazione nella Società Dante Alighieri, Roma, Bonacci, 1995; F. Cavarocchi, Avanguardie dello spirito. Il fascismo e la propaganda culturale all'estero, Roma, Carocci, 2010, pp. 167-176.

19. Cfr. la documentazione in ACS, Ministero della Cultura popolare (da ora in poi MCP), DGSP, b. 69, f. «La "Dante Alighieri" in Francia».

20. Cfr. i materiali in ACS, MCP, Gabinetto, b. 91. 
21. Cfr. ACS, MCP, DGSP, b. 72, f. «Scuola di lingua e cultura italiana». Sul ruolo di Hauvette e sull'Union intellectuelle franco-italienne, J. Dubois, L'enseignement de l'italien en France 1880-1940. Une discipline au cœur des relations franco-italiennes, Grenoble, Ellug, 2015, pp. 345-349.

22. Una prima ricostruzione in E. Decleva, Relazioni culturali e propaganda negli anni '30: $i$ comitati «France-Italie» $e$ «Italia-Francia», in J. B. Duroselle e E. Serra (a cura di), Il vincolo culturale fra Italia e Francia negli anni Trenta e Quaranta, Milano, Angeli, 1986, pp.108-157. Come ha sottolineato Decleva, il comitato gemello fondato in Italia ebbe una visibilità molto minore e si dedicò soprattutto all'organizzazione di ricevimenti ufficiali di personalità francesi in visita nella penisola.

23. «Comité France-Italie. Annuaire», 1930, p. 3.

24. Ivi, pp. 5-10.

25. «Comité France-Italie. Annuaire», 1935-1936, pp. 25-74.

26. Sul ruolo dei Comités France-Italie nell'organizzazione dei viaggi in Italia, C. Poupault, À l'ombre des faisceaux. Les voyages français dans l'Italie des chemises noires (1922-1943), Roma, École française de Rome, 2015, in specie pp. 601-708.

27. La vie du Comité, «France-Italie. Revue mensuelle», marzo-aprile 1935, p. 3.

28. Sulle origini dell'Union médicale latine, I. Renaudet, Diffusion des pratiques médicales et stratégies de promotion professionnelle à travers l'exemple de la Revue de l'Union médicale franco-ibéro-américaine (1912-1940), «El Argonauta español», nº 8, 2011, disponibile online all'indirizzo <http:// journals.openedition.org/argonauta/169>.

29. J. Dubois, L'enseignement de l'italien en France, cit., pp. 352-371; Ministero degli affari esteri, Direzione generale degli italiani all'estero, Annuario delle scuole e delle istituzioni, cit.

30. Una sintetica documentazione è conservata in ASMAE, Archivio scuole (1925-1945), b. 33, f. «Istituto di cultura italiana Francia. Gestione amministrativa». Per un inquadramento, F. Cavarocchi, Avanguardie dello spirito, cit., pp. 176-184.

31. Sulla mostra e in generale sul ruolo dei peintres italiens de Paris, che nel 1933 dettero vita a una sezione del Sindacato nazionale fascista belle arti, L. Piccioni, Les Italiens de Paris ou comment des défenseurs de l'«italianité" devinrent des ambassadeurs du "réalisme magique méditerranéen", in C. Fraixe, L. Piccioni e C. Poupault (a cura di), Vers une Europe latine. Acteurs et enjeux des échanges culturels entre la France et l'Italie fasciste, Bruxelles, Peter Lang, 2014, pp. 189-192 e passim.

32. Cfr. all'interno dell'ampia bibliografia sul ruolo politico del futurismo i saggi raccolti in $R$. De Felice (a cura di), Futurismo, cultura e politica, Torino, Fondazione Giovanni Agnelli, 1988.

33. Una ricostruzione dei preparativi e dell'esito della mostra in B. Garzarelli, «Parleremo al mondo intero». La propaganda del fascismo all'estero, Alessandria, Edizioni dell'Orso, 2004, pp. 117-125; cfr. anche E. Decleva, Relazioni culturali e propaganda negli anni '30, cit., pp. 130-134.

34. La sezione italiana al «Salon international du livre d'art», «La Nuova Italia», 26 maggio 1931, p. 3; M. Carli, Riprodurre l'Africa romana: ipadiglioni italiani all'Exposition coloniale internationale, Parigi 1931, «Memoria e ricerca», $\mathrm{n}^{\circ}$ 17, 2004, pp. 211-232.

35. Sulla figura di Fiumi, A. Giladi, Latinité et échanges intellectuels franco-italiens dans l'entre-deuxguerres. L'action de Lionello Fiumi, in C. Fraixe, L. Piccioni e C. Poupault (a cura di), Vers une Europe latine, cit., pp. 131-141.

36. Cfr. all'interno di un'ampia bibliografia L. Fiumi e A. Henneuse (a cura di), Anthologie de la poésie italienne contemporaine, Parigi, Les Écrivains réunis, 1928, trad. di E. Bestaux, P. Guiton, M.Y. Lenoir, H.-F. Marchand, A. Mortier, M. Muret, P. de Nolhac e É. Schneider; L. Fiumi e E. Bestaux (a cura di), Anthologie des narrateurs italiens contemporains, Parigi, Delagrave, 1933; B. Mussolini, Mon journal de guerre, Parigi, Éditions du Cavalier, 1932, trad. di E. Bestaux; A. Aniante, Italo Balbo, maréchal de l'air, Parigi, Grasset, 1933, trad. di F. Hayward.

37. T. Syrjämaa, Visitez l'Italie. Italian State Tourist Propaganda abroad 1919-1943. Administrative Structure and Practical Realization, Turku, Turun Yliopisto, 1997, pp. 81, 396 e passim. 
38. Ampia documentazione in ACS, MCP, DGSP, bb.68-90. Sull'attività dispiegata durante la guerra d'Etiopia, B. Garzarelli, «Parleremo al mondo intero», cit., pp. 137-143.

39. Sull'attività dei Caur e di altri mediatori fascisti in Francia, M. Cuzzi, L'Internazionale delle Camicie nere. I CAUR 1933-1939, Milano, Mursia, 2005, pp. 121-123, 176-179, 222, 241-245 e passim; B. Garzarelli, «Parleremo al mondo intero», cit., pp.91-96, 127-137; numerose indicazioni in C. Poupault, À l'ombre des faisceaux, cit.

40. Sulla controversia storiografica suscitata dal volume di Zeev Sternhell (Ni droite, ni gauche. L'idéologie fasciste en France, Parigi, Seuil, 1983), si rimanda a B. Jenkins (a cura di), France in the Era of Fascism. Essays on the French Authoritarian Right, New York-Oxford, Berghahn Books, 2005; S. Berstein e M. Winock (a cura di), Fascisme français? La controverse, Parigi, CNRS, 2014. Sulle acquisizioni di una prospettiva transnazionale, cfr. almeno D. Orlow, The Lure of Fascism in Western Europe. German Nazis, Dutch and French Fascists, 1933-1939, Basingstoke, Palgrave Macmillan, 2009; A. Bauerkämper e G. Rossoliński-Liebe (a cura di), Fascism without Borders. Connections and Cooperation between Movements and Regimes in Europe from 1918 to 1945, New York-Oxford, Berghahn Boooks, 2017; Á. Alcalde, War Veterans and Fascism in Interwar Europe, Cambridge, Cambridge University Press, 2017.

41. Sul manifesto, firmato da 850 personalità molte delle quali già attivamente impegnate nelle iniziative italo-francesi, cfr. J.-F. Sirinelli, Intellectuels et passions françaises. Manifestes et pétitions au XX ${ }^{e}$ siècle, Parigi, Fayard, 1990, pp. 92-95; C. Poupault, À l'ombre des faisceaux, cit., pp. 679-680, a cui si rimanda per le numerose e puntuali indicazioni sulle personalità coinvolte. Sull'immagine del fascismo nella stampa francese ancora indispensabile P. Milza, L'Italie fasciste devant l'opinion française, Parigi, Colin, 1967.

42. Fra i contributi sull'elaborazione dell'idea di latinità e sui suoi articolati risvolti ideologici, A. Giladi, The Elaboration of Pan-Latinism in French Intellectual Circles, from the Turn of the Nineteenth Century to World War I, «Journal of Romance Studies», vol. 14, nº 1, 2014, pp. 56-72 ; J. Guedj e B. Meazzi (a cura di), La culture fasciste entre latinité et méditerranéité (1880-1940), «Cahiers de la Méditerranée», $\mathrm{n}^{\circ}$ 95, 2017.

43. Sull'uso interno della categoria di latinità, correlato ma non coincidente con quella di romanità, cfr. ad es. L. Sorrento, Latinità, in Dizionario di politica a cura del Partito nazionale fascista, vol. 2, Roma, Istituto della Enciclopedia Italiana, 1940, pp.713-718. Un'utile messa a punto in J. Guedj e B. Meazzi (a cura di), La culture fasciste entre latinité et méditerranéité, cit.

\section{RIASSUNTI}

Il contributo ricostruisce i tentativi di inquadramento degli emigrati intrapresi dal regime fin dagli anni Venti, con l'obiettivo di rafforzarne il vincolo identitario con la madrepatria e di promuoverne la politicizzazione in senso fascista. Il governo italiano prestò parallelamente attenzione a un altro settore di intervento, ovvero le iniziative culturali e di propaganda rivolte al pubblico francese, volte a presentare l'Italia come un paese moderno e affidabile. Queste iniziative si giovarono di una serie di interlocutori francesi interessati all'esperimento fascista e cercarono di utilizzare la tradizionale rete di relazioni intellettuali e artistiche franco-italiane. La strategia fascista si affidò dunque alla costruzione di una complessa trama di attori formali e infornali; essa non fu priva di efficacia, contribuendo ad alimentare l'atteggiamento benevolo 
verso la 'sorella latina' espresso da settori non marginali dell'opinione pubblica francese ancora nei tardi anni Trenta.

L'article reconstruit les tentatives du régime depuis les années vingt pour encadrer les émigrés dans le but de renforcer leur lien identitaire avec l'Italie et de promouvoir leur politisation à l'aune de l'idéologie fasciste. Le gouvernement mussolinien intervint également dans le domaine des initiatives culturelles et de propagande destinées au public français, qui visaient à présenter l'Italie comme un pays moderne et fiable. Ces initiatives bénéficièrent d'une série d'interlocuteurs français intéressés par l'expérience fasciste et essayèrent d'utiliser la tradition des relations intellectuelles et artistiques franco-italiennes. La stratégie fasciste reposait donc sur la construction d'un réseau complexe d'acteurs formels et informels; elle ne fut pas sans efficacité, contribuant à l'attitude bienveillante envers la «sœur latine » exprimée par des secteurs significatifs de l'opinion publique française jusqu'à la fin des années trente.

\section{INDICE}

Mots-clés : fascisme, réseaux intellectuels, latinité, propagande, relations culturelles internationales

Parole chiave : fascismo, reti intellettuali, latinità, propaganda, relazioni culturali internazionali

\section{AUTORE \\ FRANCESCA CAVAROCCHI}

Université de Florence

Francesca Cavarocchi est docteure en histoire européenne à l'université de Bologne. Elle est actuellement chargée de cours en histoire contemporaine à l'université de Florence. Ses recherches portent sur l'antisémitisme en Italie, sur la politique étrangère fasciste, sur la mémoire du fascisme en Europe, sur la protection du patrimoine culturel dans l'Italie du XX ${ }^{\mathrm{e}}$ siècle. 